\title{
A Novel and Pragmatic Protocol for the Regression of Lumbar Disc Pathologies
}

\author{
Keramat Ullah Keramat ${ }^{1}$, Abdul Haseeb Bhutta ${ }^{2}$, Fayaz Ahmad ${ }^{3}$, Mian Ubaidullah Bilal ${ }^{4}$, Ayesha Junaid \\ ${ }^{1}$ Associate Professor, ${ }^{2-3}$ Senior Lecturer, ${ }^{4-5}$ Lecturer, \\ ${ }^{1-5} \mathrm{Helping}$ Hand Institute of Rehabilitation Sciences Mansehra
}

Author's Contribution

1,4-5 Conception and design, Collection and assembly of data, ${ }^{2-3} \mathrm{Analysis}$ and Interpretation of the data, ${ }^{1-2}$ Critical revision of the article for important intellectual content, Statistical expertise ${ }^{1}$ Final approval and guarantor of the article.

Article Info.

Received: Apr 28, 2020

Acceptance: Dec 30, 2020

Conflict of Interest: None

Funding Sources: None

Address of Correspondence

Dr Keramat Ullah Keramat

karamatjee@yahoo.com

Cite this article as: Keramat $\mathrm{KU}$, Bhutta AH, Ahmad F, Bilal MU, Junaid A. A Novel and Pragmatic Protocol for the Regression of Lumbar Disc Pathologies. JRCRS. 2021; $\quad 9(1)$. 3-9. DOI: https://dx.doi.org/10.53389/JRCRS.20 $\underline{21090101}$
A B S T R A C T

Background: Disc pathologies constitute the major component of the low back pain spectrum. The effects of novel and pragmatic interventions are not known.

Objective: To explore the effects of a novel and pragmatic set of treatment protocols for the management of the Lumbar disc pathologies.

Methodology: This single group quasi-experimental design recruited 16 patients of a mean $( \pm S D)$ age of $38.63 \pm 11.20$ years and mostly males $(n=14)$, of disc pathology of left of $50 \%$, right $37 \%$ and both side $13 \%$ at HHIRS Mansehra. The participants were screened against the inclusion and exclusion criteria. A consecutive sampling technique was used. Visual analogue scale (VAS) for pain at rest and mobility, Straight leg raise angle (SLR) and Ronald Morris Disability (RMD) were the outcomes measuring tools. Keramat's novel and pragmatic techniques were the main interventions.

Results: Significant $(p<0.05)$ and clinically meaningful profound improvement was observed for improvement in mean $\pm S D$ in VAS at rest of $81( \pm 19.93) \mathrm{mm}$, VAS at mobility of 60.31 $( \pm 27.37) \mathrm{mm}, \mathrm{RMD}$ of $35( \pm 12.54)$, and SLR of $32.27( \pm 19.27)$, using a paired t-test. Almost all $(n=14)$ the participants became asymptomatic with respect to pain at rest after the first session. Their constant pain became intermittent pain with mobility and in the standing position. Loss of sleep at night improved in total of 8/8 participants. RMD scores showed steady improvement over the period.

Conclusion: The novel techniques are effective in improving the pain at rest in lumbar disc prolapse cases and moderately effectively in improving the disability. However, the results cannot be generalized due to the absence of a control group.

Keywords: Lumbar disc regression, Manual therapy, Novel techniques, Physiotherapy.

\section{Introduction}

Back pain is associated with huge economical costs and is the most frequent complaint among musculoskeletal disorders. ${ }^{1}$ Disc pathologies constitute a large proportion of the back-pain spectrum. ${ }^{2}$ The statistics in Pakistan conform to the global statistics. ${ }^{2,} 3$ Pakistan is a developing country and most of its population is reliant on manual techniques for lifting weights. ${ }^{3}$ No regulation exists to make manual handling training mandatory for the laborers through their respective employers.

The most common management for prolapsed disc is unnecessary surgeries despite the fact that conservative management and surgical management yield similar outcomes in the long run. ${ }^{4,5}$ In addition, reports suggest that recurrence of the disc pathologies are not uncommon after surgical interventions. ${ }^{6}$

The etiology of the disc prolapse is the development of positive pressure at the level of the nucleus pulposus in a disc. In a flexed position, the direction of the generated force in the nucleus pulposus is in posterior or posterolateral, since the posterior margin of the vertebral endplates are distracted and anterior endplates approximated. ${ }^{7,} 8 \mathrm{~A}$ tear in the annulus fibrosis developed either gradually or suddenly due to a sudden overload or repetitive loading. ${ }^{7}$ The lordotic curvature of 
the lumbar spine in erect posture ensures to direct the maximum force anteriorly since posterior margin of the lumbar vertebrae are in an approximated position about the anterior margins. ${ }^{9-11}$ Conversely, the straightening of the lumbar spine reverses the direction of the force and predisposes to disc prolapse. 8,12

The Cyriax techniques are generalized and its focus is a generalized mobilization of the spine through mobilization and manipulation techniques. ${ }^{13}$ McKenzie's techniques are largely reliant on the gradual and graded extension from the flexed position of the spine and believe that the regression of the disc is secondary to these positions. ${ }^{14}$ Maitland's (concept) approach is more localized and concentrates on a segmental level. Posteroanterior (PA) glides are applied to the segments for mobilization of the segments via its 4 grades. ${ }^{15}$ Brian Mulligans Techniques are not specific and focuses on natural apophyseal glides (NAGS) and sustained natural apophyseal glides through it concave-convex rule. ${ }^{16}$ These schools of thought address the mobility issue but seldom comment on the mechanism of disc regression.

The creation of negative pressure within the disc $^{8}$ through pragmatic manual intervention and the subsequent effects on the restoration of the lumbar and cervical spine lordosis- and regression are not studied. Based on this principle, manual therapy protocols have been designed and tested. The current study reports the preliminary findings.

\section{Methodology}

This quasi-experimental study of pre-post design recruited 16 patients through consecutive sampling. The eligibility criteria were patients who had MRI confirmed disc prolapse with back pain radiating below the knee, age below 60 years and a detailed assessment finding consistent with lumbar disc pathology. The exclusion criteria were comorbid condition contraindicated for manual therapy such as long-term steroid use, vertebrae fracturs and previous history of CA. The study was approved by the research and ethics committee of HHIRS and rooted in the daily practice of HHIRS. Informed consent was taken from all patients. Patients were recruited from October 2019 to January 2020 (4month duration).
Convenient consecutive sampling techniques were employed. The participants were screened against the inclusion and exclusion criteria and a detailed assessment was carried out. Findings were shared with the patients and were informed of the interventions. The majority of the participants were referred by their acquaintances who had availed the services for their lumbar disc prolapse. Written consent was taken for their participation in the study.

A detailed assessment was carried of each patient meeting the inclusion criteria. Subjective information regarding the onset, behaviors of pain, nature of symptoms, aggravating factors, easing factor, previous treatments, and investigations were carried out. The Objective examination included inspection and observation of the back. Active range of motion movement, myotomal strength, sensation in dermatomes, reflexes, sacroiliac and hip joint screening. Straight leg raise (SLR) test was performed and the range was measured. Passive accessary intervertebral movement and passive intervertebral movement assessments were part of the examination process in relation to the disc pathology.

Roland-Morris low back pain and disability questionnaire (RMD) was used to assess the quality of life before the treatment and after the termination of the treatment and has been shown with good validity and reliability ${ }^{17}$. The score ranges from $0-24$ and percent scores were computed for each individual. Higher scores represent a higher disability.

Visual analogue scale (VAS) was used at bassline and after the completion of treatment sessions. It is a ratio scale and is used for both clinical as well as experimental studies which involve measurement of pain. ${ }^{18}$ It is composed of $0-100 \mathrm{~mm}$ straight-line where zero indicates no pain and $100 \mathrm{~mm}$ indicates severe pain. ${ }^{18-20}$ VAS scores were collected separately for pain at rest and pain during mobility before and after the treatment sessions.

SLR was measured through a digital inclinometer (clinometer) installed on a Samsung note 8 android phone at the initial assessment and at the final treatment. Good reliability and validity of the digital inclinometer have been reported. ${ }^{21}$ 
The following interventions were used in this study.

A- Novel and pragmatic intervention are based on the principle of negative pressure generation at the disc. ${ }^{8}$

The following are considered for the generation of the negative pressure

1- Approximation of the spinous process

2- The Distraction of the anterior intervertebral endplates

3- Approximation of the posterior intervertebral endplates

4- A sustained force or oscillation for 10 seconds with or without deep breathing

To achieve the above, a force is applied over the spinous process through the pisiform of the hand. Figure 1 shows the resolution of the applied force into 3 components.

1- The caudal component of the force is used for the approximation of the spinous process of the affected segment. The approximation of the segments is beyond the appreciation of the therapist and is based on the subjective feeling of the therapist. Similarly, the magnitude of the force cannot be determined but would equate to the magnitude of force required for grade 2 (Cyriax's mobilization). Unlike Maitland's oscillation techniques and other schools of thoughts, the applied force stabilizes the segment rather than mobilization. Deep inspiration of the diaphragm allows a good feel of the spinous process where they tend to separate. This direction of the applied force is intended to reverse or stop or hinders the natural movement of the spinous process.

2- The lateral component of the force is used if a posterolateral disc prolapse is suspected. The lateral force component widens the foramen and reduces the tension on the nerve root. A lateral component of the applied force may be skipped in the case of central disc pathology.

3- The posteroanterior component of the applied force serves to distract the anterior intervertebral end plate and approximate the posterior endplates; thereby creating a negative pressure that might serve to regress the protruded nuclear material. This is depicted in figure 2. A bio mechanical verification through appropriate model may be needed to verify this theory.

The caudal force component takes the lion's share followed by the lateral force component and finally the posteroanterior component. It is worth noting that the anterior force is applied at the end of the expiration to allow the maximum distraction of the vertebrae.

The technique is applied in each of the positions recommended by Mckenzie with modification. Lateral flexion or traction to the segments are applied if the patient is not getting asymptomatic. The techniques are administered initially in a flexed position with three pillows under the belly and gradually extended. The three pillows constitute a height from $10-20 \mathrm{~cm}$. The patient's symptoms relief guides the therapist and if the symptoms recur, the patient is brought back into the preceding position, and administration of the techniques are continued until relief is gained in the next position. A stay in one position roughly takes $5-10$ minutes. The patient is supported in all these positions and which are passive unlike the original McKenzie's positions. The passive extension practically relaxes the erector spinae muscles which allows for manual adjustments.

The general guide for the administration of the techniques is 10 seconds sustained pressure or 10 oscillations since the patient can easily hold breath between the deep inspiration and deep expiration. Three sets of interventions in each position are practiced. The sequence followed in this relation is from the fully flexed position of the spine to the fully extended position. Change in each position is brought about by reducing one pillow under the waist until neutral position is achieved and then by increasing putting adding one pillow under the chest to bring the patient into the extension. A longer duration of sustained pressure or oscillation may allow fluid accumulation around the nucleus pulposus and the negative pressure generation may be lost which in turn would serve undesirable effects.

B- Transcutaneous electrical nerve stimulation (TENS) of 30 minutes of frequency ranges from $2-10 \mathrm{~Hz}$ for the generation of endogenous opioids

C- Therapeutic Ultrasound for 5 minutes 1:1 interrupted over the effective segment for reduction of Edema and healing 
D- Core stability exercise or complete bed rest depending on the acuteness of symptoms

The interventions were administered by the principal investigator at the first session and later on by the team of professionals trained in these techniques.

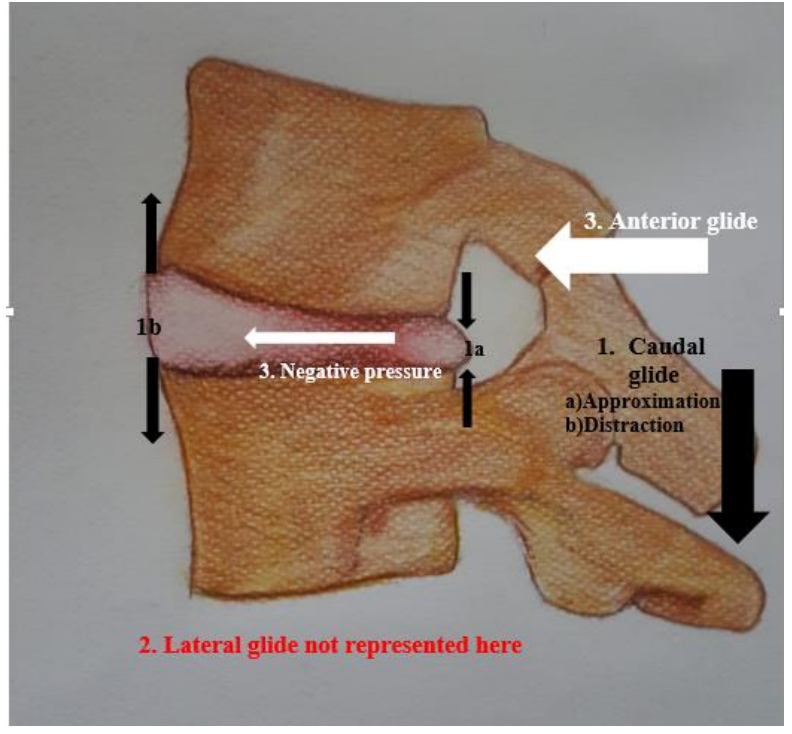

Figure 1. Applied force and expected results

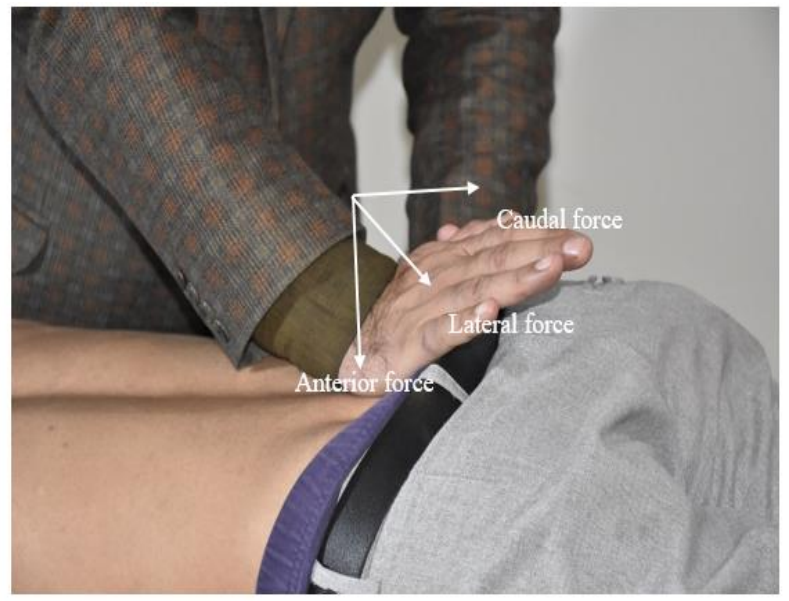

Figure 2: Resolution of the applied force in 3 components

The Statistical Package for Social Science (version 21) software was used for data analyses. Frequencies and percentages were computed for the nominal and categorical variables. A difference variable $(\Delta)$ was computed for each of the RMD, VAS at rest and mobility and SLR. A Shapiro-Wilk test for these variables showed non-significant values $(P>0.05)$. Paired t-test was used to compare the means for improvement. The data file was split in relation to the duration of symptoms into four categories to track the changes in means with respect to the duration of symptoms. Levene's test of homogeneity was non-significant ( $p>0.05)$ across all variable representing changes. A One-way ANOVA was performed between the groups of duration of symptoms since the onset of symptoms. Assumptions of equal and large sample sizes for ANOVA was violated. A Robust analysis was performed. It is assumed fine since the study is exploratory concerning the novel techniques.

\section{Results}

A consecutive sample led to the recruitment of 16 subjects meeting the inclusion criteria at HHIRS Mansehra. Detail demographics of the participants are shown in table I. The majority $(n=14)$ of the participants were males and pain in majority (50\%) was referred into the left leg. Most of the participants experienced a sudden onset following lifting a weight or sneezing. The participant received $9.44( \pm 7.06)$ sessions. Two of the participants did not show up for their final appointment and the data was collected through a phone.

\begin{tabular}{|c|c|c|}
\hline Age ( \pm SD) & Both genders & $38.63 \pm 11.20$ \\
\hline & & $\mathrm{N}(\%)$ \\
\hline \multirow{3}{*}{ Side effected } & Right & $6(37.5 \%)$ \\
\hline & Left & $8(50 \%)$ \\
\hline & Bilateral & $2(12.5 \%)$ \\
\hline \multirow[t]{2}{*}{ Gender } & Male & $14(87.5 \%)$ \\
\hline & Female & $2(12.5 \%)$ \\
\hline \multirow[t]{2}{*}{ Onset } & Sudden & $7(43.8 \%)$ \\
\hline & Gradual & $9(56.3 \%)$ \\
\hline \multirow{4}{*}{$\begin{array}{l}\text { Duration of } \\
\text { symptoms } \\
\text { since onset }\end{array}$} & Less than 10 days & $7(43.8 \%)$ \\
\hline & 11 to 20 days & $2(12.5 \%)$ \\
\hline & 20 to 30 days & $3(18.8 \%)$ \\
\hline & More than 30 days & $4(25 \%)$ \\
\hline \multirow{4}{*}{$\begin{array}{l}\text { Types of Disc } \\
\text { Pathology }\end{array}$} & Bulge & $10(62.5 \%)$ \\
\hline & Protrusion & $2(12.5 \%)$ \\
\hline & Sequestration & $2(12.5 \%)$ \\
\hline & Herniation with sequestration & $2(12.5 \%)$ \\
\hline \multirow{3}{*}{$\begin{array}{l}\text { Nature of } \\
\text { symptoms }\end{array}$} & Constant & $9(56.3 \%)$ \\
\hline & Intermittent & $7(43.8 \%)$ \\
\hline & First time & $4(25 \%)$ \\
\hline \multirow{2}{*}{$\begin{array}{l}\text { Previous } \\
\text { episodes of } \\
\text { Back pain }\end{array}$} & Once before & $11(18.3 \%)$ \\
\hline & $\begin{array}{l}\text { Two and more than two } \\
\text { episodes }\end{array}$ & $1(6.3 \%)$ \\
\hline
\end{tabular}

Paired sample t-test statics shown in table II, revealed a major change in the score of VAS at rest. 


\begin{tabular}{|c|c|c|c|c|}
\hline & $\begin{array}{l}\text { Duration of } \\
\text { Symptoms }\end{array}$ & Mean $\pm S D$ & $F$ & P-value \\
\hline \multirow[t]{5}{*}{$\overline{\Delta \mathrm{RMD}}$} & A & $39.44 \pm 7.44$ & \multirow[t]{5}{*}{0.574} & \multirow[t]{5}{*}{0.643} \\
\hline & B & $35.45 \pm 14.77$ & & \\
\hline & C & $31.96 \pm 10.46$ & & \\
\hline & $\bar{D}$ & $29.30 \pm 20.77$ & & \\
\hline & Total & $35.00 \pm 12.54$ & & \\
\hline \multirow{5}{*}{$\begin{array}{l}\text { SVAS } \\
\text { Mobility } \\
(\mathrm{mm})\end{array}$} & $\mathrm{A}$ & $67.57 \pm 23.92$ & \multirow[t]{5}{*}{-1.134} & \multirow[t]{5}{*}{0.375} \\
\hline & B & $81.00 \pm 4.24$ & & \\
\hline & $\bar{c}$ & $50.00 \pm 29.05$ & & \\
\hline & $\bar{D}$ & $45.00 \pm 34.70$ & & \\
\hline & Total & $60.31 \pm 27.37$ & & \\
\hline \multirow[t]{5}{*}{$\overline{\Delta \mathrm{SLR}}$} & $A$ & $38.67 \pm 23.96$ & \multirow[t]{5}{*}{0.529} & \multirow[t]{5}{*}{0.671} \\
\hline & B & $30.00 \pm 000$ & & \\
\hline & $\bar{C}$ & $21.66 \pm 20.20$ & & \\
\hline & D & $30.17 \pm 14.96$ & & \\
\hline & Total & $32.27 \pm 19.29$ & & \\
\hline \multirow{5}{*}{$\begin{array}{l}\triangle \text { VAS } \\
(\mathrm{mm})\end{array}$} & A & $78.57 \pm 22.11$ & \multirow[t]{5}{*}{0.879} & \multirow[t]{5}{*}{0.479} \\
\hline & B & $89.00 \pm 7.071$ & & \\
\hline & C & $70.00 \pm 29.30$ & & \\
\hline & $\bar{D}$ & $92.75 \pm 7.80$ & & \\
\hline & Total & $81.81 \pm 19.93$ & & \\
\hline
\end{tabular}

SLR: Straight Leg Raise; VAS: Visual Analogue Scale; RMD: Ronald Morris Disability Score, VAS scores in millimeters (mm); $A=$ Less than 10 days; B: 11-20 days; C: 21 to 30 Days; D: More than 30 Day

Significant $(p<0.05)$ and clinically meaningful profound improvement was observed across the change in means $( \pm S D)$ of VAS at rest $81( \pm 19.93) \mathrm{mm})$ and mobility of $60.31( \pm 27.37) \mathrm{mm}, \mathrm{RMD}$ of $35( \pm 12.54)$ and SLR of $32.27( \pm 19.27) \%$. Almost all the participants became asymptomatic with respect to pain at rest after the first session. Their constant pain became intermittent pain with mobility and in the standing position. Loss of sleep at night improved in a total of 8/8 in participants.

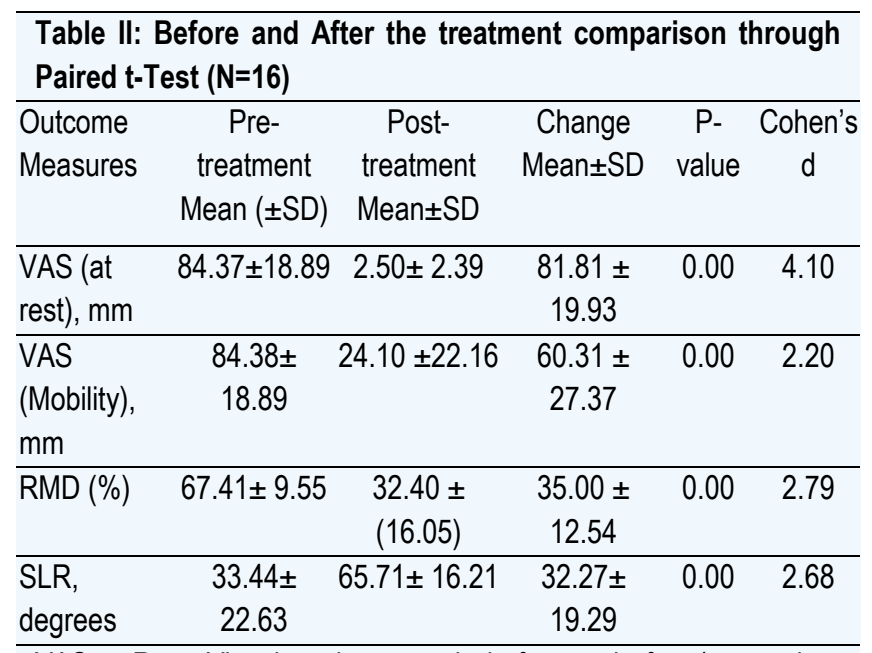

VAS at Rest: Visual analogue scale before and after 1st session; VAS mobility: Score before 1st session and after the last session;
SLR: Straight leg raise angle before 1st session and at the end of the last treatment session; RMD: Ronald Morris disability score before 1st treatment session and after the last treatment session.

One-way ANOVA results showed no significant improvement in between the groups with respect to the duration from onset of symptoms. The sample size in our case is very small and a significant difference may be expected in a large sample size. The statistics show a linear relationship in the majority of the variable for the duration of symptoms since onset and treatment. Acute cases are likely to respond to the treatment more than chronic. Figure 3 shows this relation for RMD. More than a 1 month to 6 months duration represented 4 cases.

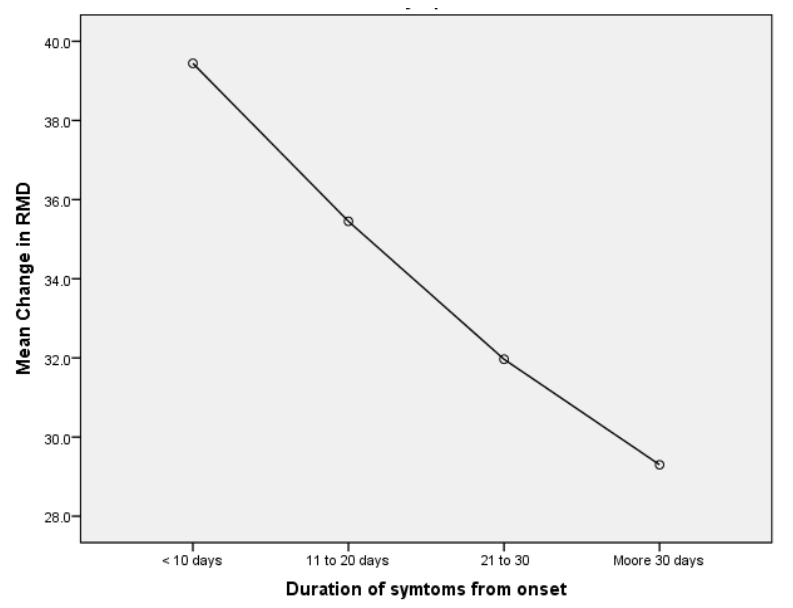

Figure 3. Linear relationship of improvement in disability score with the duration of symptoms.

\section{Discussion}

The preliminary results of this study reveal that the novel and pragmatic manual therapy techniques for the management of lumbar spine disc prolapse are effective in relieving the constant excruciating pain at rest which results from a sudden onset. Similar improvement but less profound improvements have been reported with McKenzie Exercises ${ }^{22,23}$, Mulligan exercises ${ }^{22}$, Maitland neurodynamic and mobilization with postural correction ${ }^{24,}$ ${ }^{25}$, and manual therapies in general. ${ }^{25}$ The various schools of thought address the immobility issues at the level of the facets of the spine and less emphasis is given to the mechanism of disc prolapse and strategies to reverse the mechanism in favor of disc regression.

The protocol in the current study is pragmatic and will evolve with more trial and outcomes. Through experience with previous patients and currents patients, it 
has been learned that disc pathologies need a different approach than the management of simple mechanical back pain. The majority of the disc prolapse cases on MRI and X-ray reveal straightening /or reverse lordosis of the spine. ${ }^{26,} 27$ Almost all the subjects in this study had straightening of the lumbar spine. Straightening of the spine reflects flexion at the facet level and conversely, a lordotic spine would be in extension. A recent metaanalysis reported a strong relationship between the loss of lumbar lordosis and back pain.28 The muscle spasm especially in lumbar multifidus might be secondary. Therefore, for the prolapsed disc to regress, the spine must assume lordotic posture. Less emphasis is given to restore lumbar spine through manual intervention. The technique used in the current protocol approximates the spinous process on priority and thereby generates an optimum environment for the disc regression in addition to the creation of negative pressure in the central nucleus pulposus.

The altered mechanics or lordosis helps create the negative pressure in the nucleus pulposus in addition to the approximation of the posterior intervertebral endplates which may serve to push back the protruded material as reported previously. ${ }^{8}$ The protruded nuclear material distracts the intervertebral endplates and may distract the adjacent spinous process by a fraction that cannot be appreciated through X-ray and MRI. The rupture of the annulus fibrosis and irritation of the sinovetebral nerve ${ }^{29}, 30$ in addition to the pressure on the thecal sac, inhibits the contraction of the lumbar multifidus and thereby the patient feels relatively comfortable in a flexed posture.

A pain-free position is the primary concern of the current intervention and that is why the interventions are initiated in a flexed position combined with traction and side flexion and gradual extension is restored by means of passive extension at the level of the facets.

The tear in the annulus fibrosis, irritation of the intervertebral nerve, edema and hematoma at the site of the tear are the factors that hinder instant recovery even if one anticipates full regression of the disc after the intervention. These are the factors which can presumably inhibit muscle contraction and thereby full instant recovery.
The ultrasound act as pro-inflammatory modality is mean to enhance the healing of the tear in the annulus ${ }^{31}$ and TENS machine is meant to relieve the pain originating from the annulus tear. ${ }^{32}$ Both of these modalities are not meant for the regression of the disc. ${ }^{31}$, 32 Therefore, we assume that the improvement in signs and symptoms were due to the pragmatic interventions.

The current study is of a small sample size and the findings cannot be generalized for the routine practice in physical therapy. However, further trials with a large sample size are advocated to assess the efficiency with respect to the size of the disc prolapse, age of the patient and onset of symptoms. Future studies should also include MRI confirmed disc regression after the improvement in clinical symptoms.

\section{Conclusion}

Pragmatic intervention for the management of lumbar disc prolapse are effective in reducing the initial symptoms of pain at rest dramatically. It helps to improve the quality of life and pain.

Acknowledgment: The principal investigator acknowledges the students and the therapists who took part in the collection of data.

\section{References}

1. Mok FP, Samartzis D, Karppinen J, Fong DY, Luk KD, Cheung KM. Modic changes of the lumbar spine: prevalence, risk factors, and association with disc degeneration and low back pain in a large-scale population-based cohort. The Spine Journal. 2016; 16:3241.

2. Sasi Kuppuswamy D, George JC, Chemmanam M. Prevalence of lumbar disc herniation and disc degeneration in asymptomatic Indian subjects: an MRI based study. Int. Orthop. 2017; 3:357-60.

3. Rathore FA, Rayan Attique YA. Prevalence and perceptions of musculoskeletal disorders among hospital nurses in Pakistan: a cross-sectional survey. Cureus. 2017; 9 .

4. Jacobs WC, van Tulder M, Arts M, Rubinstein SM, van Middelkoop $M$, Ostelo $R$, et al. Surgery versus conservative management of sciatica due to a lumbar herniated disc: a systematic review. European Spine Journal. 2011; 20:513-22.

5. Amundsen T, Weber H, Nordal HJ, Magnaes B, Abdelnoor $M$, Lilleås $F$. Lumbar spinal stenosis: conservative or surgical management?: A prospective 10-year study. Spine. 2000; 25:1424-36. 
6. Suk K-S, Lee H-M, Moon S-H, Kim N-H. Recurrent lumbar disc herniation: results of operative management. Spine. 2001; 26:672-6.

7. Tampier C, Drake JD, Callaghan JP, McGill SM. Progressive disc herniation: an investigation of the mechanism using radiologic, histochemical, and microscopic dissection techniques on a porcine model. Spine. 2007; 32:2869-74.

8. Keramat $\mathrm{KU}$, Gaughran A. Safe physiotherapy interventions in large cervical disc herniations. Case Reports. 2012; 2012:bcr2012006864.

9. Skaf GS, Ayoub CM, Domloj NT, Turbay MJ, El-Zein C, Hourani $\mathrm{MH}$. Effect of age and lordotic angle on the level of lumbar disc herniation. Advances in orthopedics. 2011; 2011.

10. Keramat KU, Babar MN. The effects of pragmatic posterior capsular stretch on shoulder range of motion: in press. BMJ Open sports and exercises medicine2020.

11. Pir Zada Khattak KUK, Ikram Ali. Effects of Extension, SIJ and Secondary Segment Mobilization in a Chronic Disabling Lumbar Discogenic Pain. JRCRS. 2015; 3:3.

12. Scannell JP, McGill SM. Disc prolapse: evidence of reversal with repeated extension. Spine. 2009; 34:344-50.

13. Atkins E, Kerr J, Goodlad E. A Practical Approach to Orthopaedic Medicine: A Practical Approach: Elsevier Health Sciences; 2010.

14. McKenzie R, Van Wijmen P. Treat your own back: Spinal publications New Zealand; 1985.

15. Jones MA. Clinical reasoning: From the Maitland Concept and beyond. Maitland's vertebral manipulation: Management of neuromusculoskeletal disorders. 2013; 1:14-52

16. Wilson E. The Mulligan concept: NAGS, SNAGS and mobilizations with movement. Journal of Bodywork and Movement Therapies. 2001; 5:81-9.

17. Roland M, Fairbank J. The Roland-Morris disability questionnaire and the Oswestry disability questionnaire. Spine. 2000; 25:3115-24.

18. Scott J, Huskisson E. Vertical or horizontal visual analogue scales. Ann Rheum Dis. 1979; 38:560.

19. Langley $G$, Sheppeard $H$. The visual analogue scale: its use in pain measurement. Rheumatology international. 1985; 5:145-8.

20. Carlsson AM. Assessment of chronic pain. I. Aspects of the reliability and validity of the visual analogue scale. Pain. 1983; 16:87-101.

21. Kolber MJ, Hanney WJ. The reliability and concurrent validity of shoulder mobility measurements using a digital inclinometer and goniometer: a technical report. Int $\mathrm{J}$ Sports Phys Ther . 2012; 7:306.

22. Warude T, Shanmugam S. The effect of Mckenzie approach and Mulligan's Mobilisation (SNAGS) in lumbar disc prolapse with unilateral radiculopathy. Age (years). 2014; 39:38.93-5.2.

23. Kuppusamy S, Narayanasamy R, Christopher J. Effectiveness of Mckenzie exercises and mat based pilates exercises in subjects with chronic non-specific low back pain: a comparative study. International Journal of Prevention and Treatment. 2013; 2:47-54.

24. Fatima K. Effectiveness of neurodynamics along with conventional physiotherapy for patients with prolapsed lumber intervertebral disc (plid): Bangladesh Health Professions Institute, Faculty of Medicine, the University. 2016. http://hdl.handle.net/123456789/377

25. Suri R, Panchal J. A Comparative Study between Maitland Mobilisation with Postural Correction, Postural Correction with Strengthening Exercises and Maitland Mobilisation with Strengthening Exercises in Patients with Non Specific CLBP. Indian Journal of Physiotherapy \& Occupational Therapy. 2018; 12.

26. Ansari A, Mondle MS, Hossain MA. Abnormalities in plain $\mathrm{x}$-ray findings of lumbosacral spine in prolapsed lumbar intervertebral disc. Medicine today. 2010; 22:6-11.

27. Siivola SM, Levoska S, Tervonen O, Ilkko E, Vanharanta $\mathrm{H}$, Keinänen-Kiukaanniemi $\mathrm{S}$. MRI changes of cervical spine in asymptomatic and symptomatic young adults. European spine journal. 2002; 11:358-63.

28. Chun S-W, Lim C-Y, Kim K, Hwang J, Chung SG. The relationships between low back pain and lumbar lordosis: a systematic review and meta-analysis. The Spine Journal. 2017; 17:1180-91.

29. Mahajan S, Swami AC, Kumar A. Cardiovascular changes and lumbar spine surgery: A neglected entity. Asian Journal of Neurosurgery. 2019; 14:1253.

30. PANCHAJANI R. Lumbar Disc Herniation. 2018.

31. Conner-Kerr T, Oesterle ME. Current perspectives on therapeutic ultrasound in the management of chronic wounds: a review of evidence. Chronic Wound Care Management and Research. 2017; 4:89-98.

32. Coutaux A. Non-pharmacological treatments for pain relief: TENS and acupuncture. Joint Bone Spine. 2017; 84:65761. 